\title{
Autocuidado e Adoecimento dos Homens: Uma Revisão Integrativa Nacional
}

\author{
Self-care and Men's Diseases: An Integrative National Review
}

\section{Autocuidado y Enfermedad de los Hombres: Una Revisión Integrativa Nacional}

\author{
Luis Henrique Costa Garcia
}

Universidade Luterana do Brasil

Nicolas de Oliveira Cardoso ${ }^{1}$

Pontifícia Universidade Católica do Rio Grande do Sul

\author{
Cláudia Maria Canestrine do Nascimento Bernardi \\ Faculdade São Francisco de Assis
}

\begin{abstract}
Resumo
A Política Nacional de Atenção Integral à Saúde do Homem (PNAISH) visa promover a prevenção de saúde do homem. Contudo estudos recentes apontam para a necessidade de maior diálogo relacionado aos sentimentos dos homens frente ao adoecimento e à cultura social e laboral. Dessa forma, essa revisão integrativa objetiva verificar os fatores culturais, emocionais e laborais relacionados ao processo de adoecimento e autocuidado dos homens. As buscas por artigos empíricos foram conduzidas nas bases de dados, Biblioteca Virtual em Saúde (BVS), SciELO e PePSIC. Ao término das etapas de seleção, nove estudos foram incluídos para análise nos resultados desta revisão. Os principais resultados sugerem que os homens sentem medo e vergonha de suas vulnerabilidades e do adoecimento. Também percebem uma imposição de papéis patriarcais pela sociedade, considerando o autocuidado como um atributo feminino. Observou-se ainda tendência à priorização do trabalho mesmo que isso implique o deterioramento da saúde.

Palavras-chave: saúde do homem, autocuidado, promoção da saúde, prevenção da saúde
\end{abstract}

\begin{abstract}
The National Policy of Integral Attention to Men's Health (PNAISH) aims to promote the prevention of men's health. However, recent works showed the need of more discussion related to men's feelings about diseases and the impacts in social and work culture. Thus, this paper aims to verify the cultural, emotional and labor factors related to the process of fall ill and self-care of men. The search for empirical articles was conducted in the databases, Virtual Health Library (BVS), SciELO and PePSIC. Nine studies were included for results analysis in the end of selection stages. The main findings suggest that men feel fear and shame about their vulnerabilities and illness. They also perceive an imposition of patriarchal roles by society, considering self-care as a feminine attribute. Also, there was a tendency to prioritize work even if this implies health deterioration.

Keywords: men's health, self-care, health peromotion, health prevention

\section{Resumen}

La Política Nacional de Atención Integral a la Salud del Hombre (PNAISH) objetiva promover la prevención de salud del hombre. Sin embargo, estudios recientes apuntan a la necesidad de diálogo relacionado con los sentimientos de los hombres frente a la enfermedad y la cultura social y laboral. De esta forma, esta revisión objetiva verificar los factores culturales, emocionales y laborales relacionados con el proceso de enfermedad y autocuidado de los hombres. Las búsquedas por artículos empíricos fueron conducidas en las bases de datos, Biblioteca Virtual en Salud (BVS), SciELO y PePSIC. Al término de las etapas de selección, se incluyeron nueve estudios para analize en los resultados. Los achados sugieren que los hombres sienten miedo y vergüenza de sus vulnerabilidades y de la enfermedad. También perciben una imposición de papeles patriarcales por la sociedad, considerando el autocuidado como un atributo femenino. Se observó una tendencia a la priorización del trabajo, aunque ello implique el deterioro de la salud.

Palabras clave: salud del hombre, autocuidado, promoción de la salud, prevención de la salud
\end{abstract}

\footnotetext{
${ }^{1}$ Endereço de contato: Av. Ipiranga, 6681- Partenon, Porto Alegre, RS, CEP 90619-900, Prédio 11, $9^{\circ}$ andar, sala 921. Telefone: (51) 981671820, E-mail: nicolas.deoliveira@hotmail.com
} 


\section{Introdução}

No sistema de saúde brasileiro, grande parte do atendimento prestado na atenção primária costuma ser direcionado aos grupos de pessoas classificadas como vulneráveis (Batista, Ribeiro, Ribeiro, Paula, \& Araujo, 2016). Dessa forma, existem diversos programas governamentais dirigidos para a saúde das crianças (Oliveira \& Veríssimo, 2015), mulheres (Medeiros \& Guareschi, 2009) e idosos (Silva, Mambrini, Peixoto, Malta, \& Lima-Costa, 2017).

Embora existam políticas públicas voltadas para a saúde do homem, o enfoque na importância do autocuidado com a saúde da população masculina costuma ser menos noticiado. Além disso, tal como ocorre com outras notícias relacionadas à saúde pública, quando surgem propagandas e até mesmo o desenvolvimento de políticas públicas, muitas vezes as opiniões de especialistas são priorizadas em detrimento de testemunhos e opiniões da população, neste caso os homens (Tomé \& Lopes, 2012).

Tais fatores culturais contribuem com a prevalência de desinformação entre os homens, os quais costumam acreditar que são invulneráveis (Wynter, Wilson, Thean, Bei, \& Fisher, 2018) ou que precisam exercer o papel de provedor familiar (Lima et al., 2014). O Ministério da Saúde (MS) aponta que essas crenças podem ocasionar na redução da procura por serviços de saúde contribuindo para o aumento dos gastos ao contribuinte e ao Sistema Único de Saúde (SUS), além de elevar os índices de mortalidade do gênero masculino (Brasil, 2009; Vasconcelos \& Frota, 2018).

Assim, em 2009, foi instaurada pelo governo brasileiro, como uma de suas prioridades, a Política Nacional de Atenção Integral à Saúde do Homem (PNAISH) visando promover atividades de saúde que auxiliem no entendimento das especificidades de cada homem, nos seus inúmeros contextos sociais, econômicos, políticos e culturais, além de concepções voltadas para o aumento da expectativa de vida. Resultando na diminuição das taxas de mortalidade e morbidade para causas que possam ser prevenidas e evitadas entre os 20 e os 59 anos (Brasil, 2009).

É importante apontar que, de acordo com a PNAISH, o número de óbitos, devido a doenças cardiovasculares e tumores, em homens acima dos 40 anos é muito maior do que em jovens devido a fatores externos (violência, acidentes, suicídio) (Brasil, 2009). Acredita-se que tal fato ocorra uma vez que os homens apresentem maior vulnerabilidade para o desenvolvimento de doenças graves crônicas, as quais costumam ser negligenciadas durante a juventude, fazendo com que quadros os quais poderiam ser prevenidos causem prejuízos irreparáveis. Além disso, é comum que os homens acima dos 40 anos acabem ingressando diretamente nos serviços secundários e terciários (Brasil, 2009; Vasconcelos \& Frota, 2018).

Além de sobrecarregar os serviços de atenção secundária e terciária, a negação dos homens à atenção primária sobrecarrega financeiramente a sociedade como um todo e também promove sofrimento psíquico e físico para o sujeito e seus familiares. Tal sobrecarga ao sistema de saúde ocorre devido à elevada porcentagem de homens entre 20 a 59 anos (Brasil, 2009).

Em 2009, a PNAISH estimava que os homens nessa faixa etária representavam cerca de 52 milhões de indivíduos, equivalente a $27 \%$ da população brasileira e que verdadeiramente eram quase invisíveis para os programas e estratégias de saúde pública (Brasil, 2009). Dados atuais do Instituto Brasileiro de Geografia e Estatística (IBGE) indicam que esse número 
chegou aos 58 milhões, o que atualmente equivale a $28 \%$ da população brasileira (IBGE, 2018). Com isso, observa-se a necessidade de reforçar, além de especializar, as ações na atenção primária com intenção de prevenir doenças e promover a saúde masculina (Brasil, 2009).

Enfermeiros (as) que atuam diretamente nos serviços de saúde indicam que tais serviços estão estruturados para atender o público feminino, sendo este um fator de impedimento para que os homens possam participar de maneira mais efetiva e buscar atendimento nas unidades de saúde. Além disso, a mulher vem ganhando mais espaço na sociedade, conquistando a ampliação do seu cuidado e, com isso, políticas de saúde foram estabelecidas objetivando reduzir índices de mortalidade de crianças e mulheres, entre outras ações, algo que não aconteceu para o público masculino (Moreira \& Carvalho, 2016).

Através da PNAISH, é possível que ocorram mudanças significativas nessa população, aproximando-os das unidades de Atenção Primária à Saúde (Brasil, 2009). Para isso é fundamental que os profissionais que trabalham na saúde atuem no cuidado à população masculina embasados nos princípios da universalidade, humanidade e equidade (Moreira \& Carvalho, 2016). É importante lembrar que o cuidado é um princípio fundamental do ser humano, antes mesmo que ele realize qualquer tipo de ação. Se não receber cuidado desde que nasce até o final da vida, o ser humano tende a desorganizar-se e debilitar-se (Boff, 2014).

Sendo assim, o autocuidado emerge com uma função regulatória, a qual possibilita aos indivíduos desempenharem um papel, de maneira autônoma, que promovam ações direcionadas à preservação da vida, da saúde, da evolução e do bem-estar. Sujeitos acometidos de doenças crônicas relatam que o autocuidado é um processo de transformação no que tange aos sentimentos relacionados a eles próprios e também na retomada de um sentido para suas vidas, fornecendo um motivo para caminhar em direção ao futuro (Galvão \& Janeiro, 2013).

Historicamente o processo que envolve a saúde-doença vem seguido de importante evolução, já que, na Era Moderna, a ciência estava voltada para o reducionismo, objetividade, saber fragmentado, interpretando os fatos através de formas evidenciadas, passíveis de serem calculadas e, na contemporaneidade, surge a subjetividade e a complexidade tentando decifrar a realidade com exemplos que levam em consideração as relações ajustadas ao meio em que os indivíduos vivem (Backes et al., 2009).

Apesar da tentativa de promover a saúde, na realidade, ainda estamos vinculados ao discurso de prevenção, porém centrado na doença. É necessário que os profissionais que atuam na área da saúde ultrapassem o modelo biomédico vigente, focado no adoecimento, no diagnóstico, na impessoalidade, na tecnologia e dediquem ações num novo modelo que contemple as múltiplas dimensões humanas, os aspectos biopsicossociais, sócio-históricos, reconhecendo a pluralidade cultural brasileira, acolhendo as características de cada sujeito (Backes et al., 2009). Alguns autores destacam ainda que os homens relatam que o trabalho ocupa um espaço em suas vidas que vai além da sobrevivência, sendo uma forma que o remete à preservação da sua identidade (Santos et al., 2017).

Se o trabalho tem esse nível de significância, fazendo com que os homens deixem de cuidar da própria saúde, modificações essenciais nos processos de trabalho das equipes de saúde são necessárias para que os homens tenham condições e estejam motivados para 
acessar os serviços de saúde (Santos et al., 2017). A atenção especializada nos locais de trabalho poderia auxiliar na redução de diversas doenças como estresse e depressão (Moreira \& Carvalho, 2016). Tendo em vista todo esse contexto e a importância dessa temática, este estudo objetivou verificar os fatores culturais, emocionais e laborais relacionados ao processo de adoecimento e autocuidado dos homens brasileiros.

\section{Método}

Este trabalho seguiu os passos sugeridos por Mendes, Silveira, e Galvão (2008) para a construção de uma revisão integrativa da literatura. Esses autores sugerem que uma revisão integrativa tem como objetivo reunir e sintetizar resultados de pesquisas sobre determinado tema ou questão, de uma forma categórica e ordenada, visando aprimorar o conhecimento da área escolhida.

As buscas foram realizadas, por dois juízes independentes, em dezembro de 2018, nas seguintes bases de dados eletrônicas: Biblioteca Virtual em Saúde (BVS), Scientific Eletronic Library Online (SciELO) e nos Periódicos Eletrônicos em Psicologia (PePSIC). Os descritores utilizados nessas bases foram: "Men's Health" AND "Self Care". O único filtro utilizado nas bases de dados foi o de artigos no idioma Português.

Os critérios de inclusão para seleção dos artigos foram: I) estudos publicados em idioma português brasileiro; II) artigos empíricos; III) estudos que avaliaram a percepção de homens em relação ao seu adoecimento e autocuidado. Não foram estabelecidas restrições de idade nem de tempo das publicações, buscando uma varredura mais abrangente da literatura nacional. Os critérios de exclusão utilizados foram: I) artigos duplicados; II) dissertações, teses e trabalhos de conclusão de curso não publicados; III) livros e capítulos de livros.

A coleta de dados ocorreu através da análise minuciosa dos títulos, resumos e palavras-chave dos artigos localizados nas bases de dados. Inicialmente foram excluídos os artigos que não apresentavam, em seu título ou resumo, ao menos uma das palavras-chave utilizadas, assim como os artigos duplicados.

\section{Apresentação e Discussão dos Resultados}

A busca inicial nas bases de dados retornou um total de 67 artigos. Após a aplicação dos critérios de inclusão e exclusão, foram selecionados 09 estudos para compor os resultados da presente revisão. A Figura 1 apresenta de forma detalhada o número de artigos encontrados em cada um das bases de dados, assim como as etapas de seleção dos artigos. 
Levantamento dos artigos nos bancos de dados

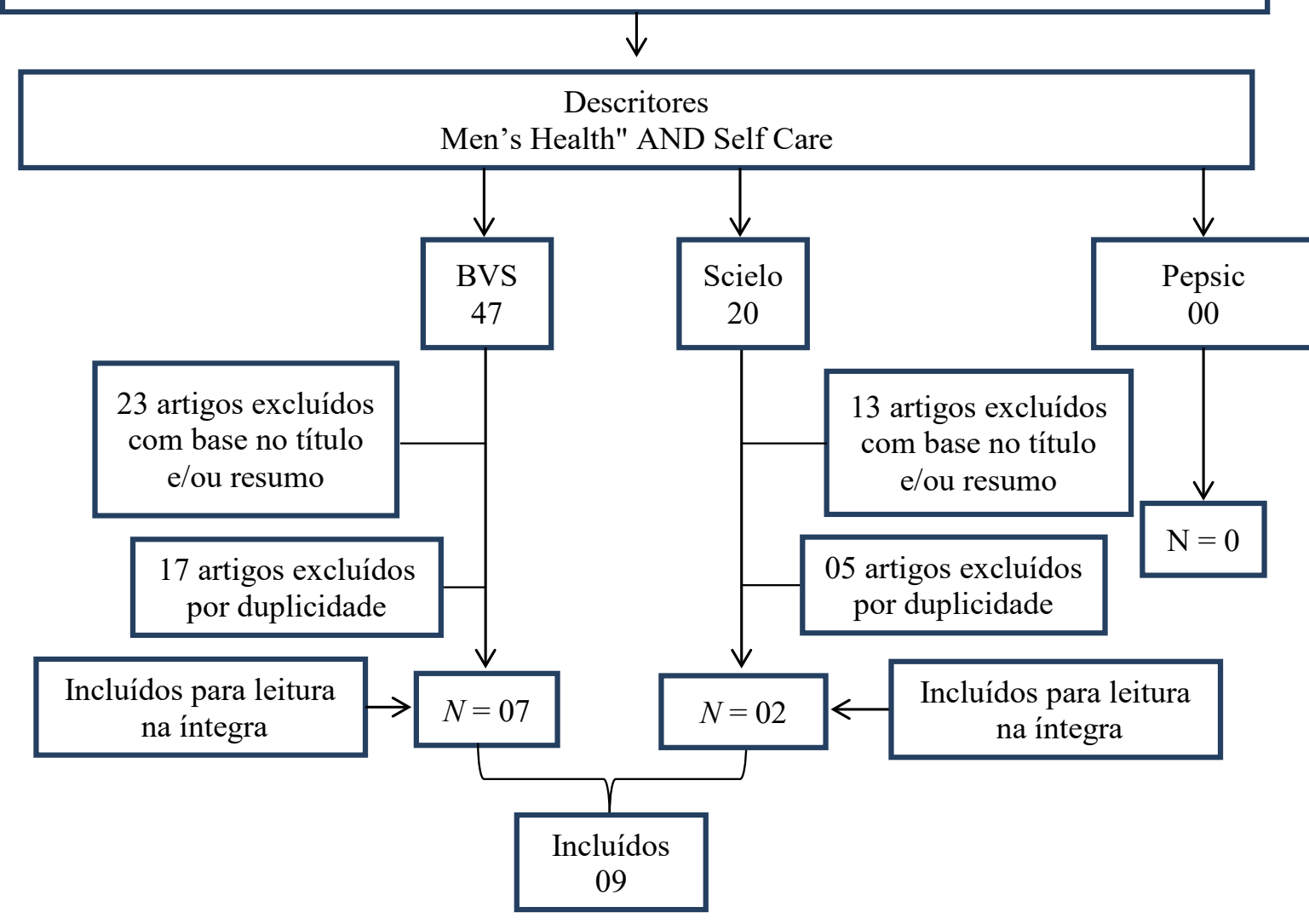

Figura 1. Fluxograma das Buscas Realizadas.

Os artigos incluídos como amostra desta pesquisa são apresentados na Tabela 1, onde estão especificadas as informações sobre: número de cada artigo, autores e ano de publicação, objetivo e método. Os números dos artigos que constam na Tabela 1 (de 0 a 8) são apresentados ao longo do texto, servindo como indicativos de referência para a apresentação e discussão dos resultados encontrados.

Tabela 1

Sinopse dos Principais Dados dos Artigos Selecionados

\begin{tabular}{|c|c|c|c|}
\hline $\mathbf{N}^{\circ}$ & Autores e ano & Objetivos & Método \\
\hline 0 & $\begin{array}{l}\text { Lemos, Ribeiro, } \\
\text { Fernandes, Bernardes e } \\
\text { Fernandes (2017) }\end{array}$ & $\begin{array}{l}\text { Identificar os motivos pelos quais } \\
\text { os homens procuram os serviços de } \\
\text { saúde. }\end{array}$ & $\begin{array}{c}\text { Qualitativo. Vinte e nove homens (31- } \\
40 \text { anos) pacientes de um Hospital no } \\
\text { município Rio de Janeiro, RJ }\end{array}$ \\
\hline 1 & Amaral et al. (2017) & $\begin{array}{c}\text { Conhecer o contexto do homem ao } \\
\text { adoecer e sobreviver ao câncer de } \\
\text { mama }\end{array}$ & $\begin{array}{l}\text { Qualitativo. Dois homens (66-74 anos) } \\
\text { sobreviventes do câncer de mama em } \\
\text { Pelotas, Rio Grande do Sul (RS) }\end{array}$ \\
\hline 2 & Sousa et al. (2016) & $\begin{array}{l}\text { Analisar a busca de homens pelos } \\
\text { serviços de Atenção Básica à Saúde e } \\
\text { sua relação com a construção social } \\
\text { das masculinidades }\end{array}$ & $\begin{array}{l}\text { Qualitativo. Dez homens (18-59 anos) } \\
\text { usuários de uma Unidade de Saúde } \\
\text { da Família (USF) em Feira de Santana, } \\
\text { Bahia }\end{array}$ \\
\hline 3 & $\begin{array}{l}\text { Viana, Costa, Santos e } \\
\text { Anjos (2015) }\end{array}$ & $\begin{array}{l}\text { Analisar a relação do homem com o } \\
\text { cuidado a sua saúde }\end{array}$ & $\begin{array}{c}\text { Qualitativo. Vinte homens (25-59 } \\
\text { anos) cadastrados em uma USF em } \\
\text { Maceió, Alagoas (AL) }\end{array}$ \\
\hline
\end{tabular}




\begin{tabular}{|c|c|c|c|}
\hline $\mathbf{N}^{\circ}$ & Autores e ano & Objetivos & Método \\
\hline 4 & $\begin{array}{l}\text { Hemmi, Conceição e } \\
\text { Santos (2015) }\end{array}$ & $\begin{array}{l}\text { Apreender as representações sociais } \\
\text { de homens sobre saúde e doença, } \\
\text { além de identificar em que momentos } \\
\text { procuram por serviços de saúde }\end{array}$ & $\begin{array}{c}\text { Qualitativo. } 20 \text { homens (20-59 } \\
\text { anos) que participam do "Terço dos } \\
\text { Homens" em Igrejas de Diamantina, } \\
\text { Minas Gerais (MG) }\end{array}$ \\
\hline 5 & $\begin{array}{l}\text { Xavier, Ceolin, Guanilo } \\
\text { e Mendieta (2015) }\end{array}$ & $\begin{array}{l}\text { Conhecer os cuidados à saúde no } \\
\text { olhar da população masculina que } \\
\text { frequenta grupos de educação em } \\
\text { saúde de uma Unidade Básica de } \\
\text { Saúde (UBS) no Sul do RS }\end{array}$ & $\begin{array}{l}\text { Qualitativo. Seis homens (35-56 anos) } \\
\text { usuários de uma UBS de Pelotas, RS }\end{array}$ \\
\hline 6 & Costa e Moura (2013) & $\begin{array}{l}\text { Identificar o significado para os homens } \\
\text { sobre o exame clínico de toque da } \\
\text { próstata para detecção precoce de } \\
\text { câncer, caracterizar a causa do déficit } \\
\text { na procura de exames preventivos e } \\
\text { serviços de saúde pelos homens }\end{array}$ & $\begin{array}{l}\text { Qualitativo. Dezessete homens (40>) } \\
\text { vinculados a uma instituição pública } \\
\text { de ensino superior, no município Rio } \\
\text { de Janeiro, RJ }\end{array}$ \\
\hline 7 & $\begin{array}{l}\text { Storino, Souza e Silva } \\
\qquad(2013)\end{array}$ & $\begin{array}{c}\text { Analisar as necessidades de saúde } \\
\text { de homens usuários de uma unidade } \\
\text { básica de saúde, na cidade de Belo } \\
\text { Horizonte }(\mathrm{BH}), \mathrm{MG}\end{array}$ & $\begin{array}{l}\text { Qualitativo. Vinte e sete homens (20- } \\
59 \text { anos) usuários de uma UBS em BH, } \\
\text { MG }\end{array}$ \\
\hline 8 & Borges e Seidl (2013) & $\begin{array}{l}\text { Verificar os efeitos de uma intervenção } \\
\text { psicoeducativa para fortalecer o } \\
\text { autocuidado entre homens idosos, na } \\
\text { busca e utilização de serviços de saúde }\end{array}$ & $\begin{array}{c}\text { Qualitativo. Treze homens (62-78 } \\
\text { anos) de um Centro de Convivência } \\
\text { para Idosos (CCI) em uma universidade } \\
\text { privada no Distrito Federal }\end{array}$ \\
\hline
\end{tabular}

Observa-se que todos os artigos utilizaram o método qualitativo através da utilização entrevistas semiestruturadas. Tal fato acaba por restringir o tamanho amostral dos estudos, contudo também permite maior riqueza em detalhes obtidos através da análise das entrevistas. Destaca-se que os métodos utilizados pelos estudos incluídos estão adequados aos seus respectivos objetivos.

Em relação à faixa etária, a amostra da maioria dos trabalhos foi composta por homens com menos de 60 anos de idade $(n=6)$. Esse achado demonstra que a literatura nacional está engajada na prevenção a saúde de homens entre os 20 e 59 anos, conforme sugerido pelo governo brasileiro (Brasil, 2009). Contudo a maior parte dos estudos $(n=6)$ foram realizados no sul e sudeste do Brasil, evidenciando que outras regiões do país ainda carecem de pesquisas voltadas ao estudo da visão dos homens frente à prevenção a sua saúde.

Tais estudos são primordiais para que seja possível pensar em estratégias de prevenção a saúde direcionadas a um público específico, sobretudo devido às diferenças culturais características das diferentes regiões do Brasil, sendo recomendado pelo Ministério da Saúde (MS) que as estratégias de prevenção ou intervenção sejam realizadas por profissionais que residam na comunidade onde atuam (Brasil, 1997).

De modo geral, os estudos buscaram compreender as relações entre a população masculina e os cuidados dirigidos à própria saúde, necessidades de saúde dos homens no contexto atual, a busca aos serviços nas unidades básicas de saúde e os sentimentos implicados no processo do adoecimento. A Tabela 2 apresenta os principais resultados encontrados nos estudos que compõem a amostra desta revisão. 
Tabela 2

Principais Resultados dos Artigos Desta Revisão

O olhar do homem para si mesmo tem representação na força, invulnerabilidade e provimen-

to. Autocuidado não é uma prática culturalmente comum dos homens. $66 \%$ dos participantes relataram procurar serviços de saúde somente quando há sintomas agudos. Impossibilidade de trabalhar induz os homens a procurarem os serviços de saúde.

J.M. apresenta visão estigmatizada dos tratamentos, temor entendido como algo horrível, dicotomia implícita do aceitar ou não o processo de adoecer, negação. O.M aceita o diagnóstico com otimismo, adapta-se à nova realidade, contudo, apresenta limitações físicas com redução da carga de trabalho. Trabalho ocupa espaço importante na vida dos homens.

Trabalho é o motivo principal pela busca tardia ao serviço de saúde. Concepção curativa de saúde, não valorizam a prevenção, só buscam assistência após agravamento da situação/doença, que os impossibilitem de trabalhar. Saúde e autocuidado fundamentadas em bases patriarcais

2 com papéis rígidos e próprios do homem, necessidade de reproduzi-los para diferenciá-los dos papéis femininos. Desconhecimento de si, do seu corpo. Cuidado à saúde é associado a atributo feminino. Visão de que mulheres pertencem ao mundo doméstico, tem tempo para cuidar da própria saúde, homem é do trabalho.

Homem é ser forte, não adoece. Doença é fragilidade. Visão curativa de saúde. Sentimentos de medo, vergonha. Reconhecimento da importância da prevenção. Negligência com a própria

3 saúde por falta de tempo e por causa do trabalho. Dinheiro e trabalho são prioridades. Medo de perder o emprego e se ausentar do trabalho. Pai de família não tem o direito de adoecer. Desconhecimento sobre política pública de saúde para homens.

Valorização da qualidade de vida, bem-estar físico, psíquico e espiritual. Felicidade atrelada à prática de esporte, boa alimentação e atividades religiosas. Prevenção é importante. Compreendem a Saúde como ausência de doença, sem saúde não a sentido para viver.

4 Entendem que doença é um mal, anomalia, desafio, vem das bactérias. Visão curativa de saúde, automedicação, busca tardia por atendimento. Autocuidado é atributo feminino. Negligência com a saúde por medo e desconhecimento das doenças e ligado às questões culturais, como machismo.

Saúde integral desde o meio ambiente até o indivíduo é importante, ausência da mesma invalida outros aspectos da vida. Tabu sobre cuidar da saúde. Prevenção promove saúde. Doença é 5 desequilíbrio do organismo, pior coisa que pode acontecer com o ser humano. Necessário ter hábitos de prevenção: alimentação adequada, consultas médicas, tomar medicamentos prescritos, participar de grupos para doentes crônicos. Qualidade de vida: necessário atividades e espaços de lazer, equilibrar trabalho, lazer, família e saúde.

Câncer de próstata é visto como algo ligado à morte. Exame do toque retal é visto como constrangedor. Nível socioeconômico maior é fator para cuidado com a saúde e busca de informa-

6 ção. Baixa adesão à prevenção está associada ao desconhecimento sobre a PNAISH. Falta de tempo e horário das UBS citados como fatores para não realizarem exame preventivo relacionado à próstata. Visão curativa de saúde.

Vínculo com profissional de saúde ou equipe auxilia na busca pelos serviços. Confiança no profissional que conhece suas necessidades/histórico. Atendimento cuidadoso é fator para

7 aumento da procura aos serviços de saúde pelos homens. Homens percebem necessidades de saúde, mas desconhecem suas vulnerabilidades. Horário de trabalho dificulta busca por prevenção/atendimento.

Participantes com pelo menos uma doença crônica grave. Principais especialidades médicas consultadas: cardiologistas e urologistas. Crença de que hábitos saudáveis e exercícios físicos

8 substituem atendimentos médicos. Crença de que realizar consultas leva ao descobrimento de doenças. Prática de automedicação, autodiagnostico. Temor do exame do toque retal. Após intervenção psicoeducativa, ocorreu aumento da frequência das consultas médicas. 
A partir da leitura dos artigos incluídos, com a intenção de verificar os fatores relacionados ao processo de autocuidado e adoecimento de homens, emergiram três grandes categorias, cada uma composta por algumas temáticas especificas. Tais categorias e temáticas são apresentadas na Tabela 3. Destaca-se ainda que os participantes dos estudos foram nomeados conforme o código de identificação utilizado nos artigos originais (e.g. H2, H3, Nego, Verde).

Tabela 3

Categorias e Temáticas Emergentes

\begin{tabular}{|c|c|c|}
\hline Categorias & Principais temas & Artigos \\
\hline 1. Fatores culturais que dificultam o autocuidado & $\begin{array}{c}\text { Autocuidado } \\
\text { Invulnerabilidade } \\
\text { Machismo } \\
\text { Saúde curativa }\end{array}$ & $0,2,3,4,5,6,7,8$ \\
\hline 2. Sentimentos manifestados & $\begin{array}{l}\text { Desconforto } \\
\text { Medo } \\
\text { Constrangimento } \\
\text { Impotência }\end{array}$ & $1,3,4,5,6,8$ \\
\hline 3. Valor do Trabalho na Vida dos Homens & $\begin{array}{c}\text { Valor } \\
\text { Utilidade } \\
\text { Masculinidade }\end{array}$ & $0,1,23,6,7,8$ \\
\hline
\end{tabular}

\section{Categoria 1 - Fatores Culturais que Dificultam Ações de Autocuidado}

Na categoria 1, os participantes dos artigos 2, 3, 4, 6 e 8 percebem o autocuidado como atributo feminino. Cuidar-se, então, é reconhecido como parte constituinte do comportamento feminino, e não do gênero masculino. A cultura de ser forte e viril remete ao pensamento de que o homem não pode adoecer e acaba não desenvolvendo o hábito de prevenção da saúde.

Além disso, todos os artigos da categoria 1 apontam que a masculinidade ainda é fortemente ligada à cultura machista. Nesta, a cultura do macho dita as regras do comportamento masculino, indicando que ele é capaz de fazer tudo sem sofrer qualquer tipo de consequência. É o ser dominante que carece de escuta, apenas determina e comunica o que quer fazer, enquadrando o homem numa espécie de máquina indestrutível, não admitindo entrar em contato com seus limites, buscando ajuda somente quando o sintoma agudo se apresenta, ou seja, a visão curativa da saúde em detrimento da preventiva.

Tais temáticas podem ser evidenciadas nos relatos de alguns participantes dos artigos analisados: "O homem sai para trabalhar, a mulher fica dentro de casa fazendo os deveres de casa e depois sempre procura o posto" (H3) (artigo 2). "O homem não cuida muito da saúde, por vários fatores: a questão do machismo, por achar que só quando está doente deve procurar a unidade, tem as palestras e ele não participa" (H8) (artigo 2). "Tenho que mostrar que sou homem. Que não adoeço, que estou forte" (Nego) (artigo 3).

Hum, só vou quando... por exemplo, se eu tomo uma pancada, eu trabalho no meu serviço, com serviço pesado. Quando eu faço uma força, um mau jeito, ou gripe. Única coisa 
que eu tenho mais de constante também é gripe. Sempre pego uma. Só quando vejo que o negócio tá pegando mesmo [. . . ]. (E3) (artigo 4).

Só vou quando há necessidade. O problema aí eu tiro pelo seguinte, quando eu vejo que algo não tá bem, por exemplo, não tô urinando suficiente, sinto dores, então eu me sinto por obrigação ir lá fazer alguns exames. (Franco) (artigo 8).

Esses achados corroboram estudos anteriores, os quais apontam que para os homens a relação com o autocuidado está permeada por crenças patriarcais, estruturadas como correntes rígidas de masculinidades que ditam os papéis a serem reproduzidos pelos homens, diferenciando-os dos papéis sociais das mulheres (Silveira, Melo, \& Barreto, 2017). Os homens seguem com a crença de que não faz sentido procurar algo que possa estar errado com a sua saúde quando na verdade não apresentam sintomas ou sinais, contribuindo para que fiquem expostos a situações que podem se agravar e que poderiam ser detectadas antecipadamente.

Essas crenças estão intimamente atreladas à cultura e às interações sociais, sobretudo a noção de invulnerabilidade masculina e a necessidade de ser o provedor familiar (Silveira et al., 2017). Por consequência, a cultura machista contribui para que os homens não busquem atendimento em saúde, pois o modelo hegemônico de masculinidade, que até hoje predomina, dificulta que eles tenham atitudes direcionadas para o autocuidado (Silveira et al., 2017).

Sendo assim, é imprescindível a necessidade de mudança na mentalidade da população masculina, para que possam aceitar a realização de hábitos relacionados ao autocuidado (Silveira et al., 2017). Contudo é importante observar a necessidade de capacitação dos profissionais da área da saúde dentro do contexto cultural dos homens em suas respectivas localizações geográficas, visto que os serviços de saúde ainda não estão organizados para atender as demandas desse público (Brasil, 1997; Viana et al., 2015).

\section{Categoria 2 - Sentimentos Manifestados}

Nessa categoria, serão apresentados os sentimentos expressos pelos homens quando os temas se referem à saúde ou mesmo quando já estão adoecidos. Os artigos 1, 3, 4, 5, 6 e 8 sugerem que, pelo fato de não considerarem a possibilidade de adoecer, a população masculina sente desconforto, medo e constrangimento quando necessitam realizar exames, consultar um profissional da saúde ou passar pelo processo de uma eventual internação hospitalar.

Todos os artigos da categoria 2 apontam que a cultura machista exerce influência nos padrões de comportamentos e emoções masculinas. Sendo assim, adoecer, necessitar de ajuda com a saúde que se debilitou é percebido por eles próprios e por alguns membros da sociedade, como sinal de impotência, de fracasso, algo não previsto que desperta temor e incertezas.

O medo e a vergonha são destacados nos artigos 1 e 3 quando é lembrado que os homens são reprimidos socialmente em relação a reconhecer fragilidades e manifestar emoções. Com isso, a procura por um serviço de saúde passa a ser um movimento que os remete à sua fragilidade. 
Tais emoções podem ser observadas nos relatados de alguns dos participantes: "Não, não precisei fazer nada disso daí (quimioterapia e radioterapia) graças a Deus, é horrível aquilo" (J. M., 74 anos) (artigo 1). "Medo. É medo de você. . . descobrir alguma doença grave... às vezes tenho medo de descobrir que estou com câncer" (Nego) (artigo 3). "A maioria dos homens têm receio sim, tem vergonha, se tem alguma coisa, tem medo. . . principalmente 0 exame de próstata" (Verde) (artigo 3).

No artigo 6, dos dezessete homens que participaram da pesquisa, 52,9\% relataram constrangimento no que se refere ao exame do toque preventivo ao câncer de próstata. Tal dado é reforçado pelo artigo 8, o qual aponta que o exame do toque retal foi relatado por sete participantes de um total de treze homens idosos, como sendo o procedimento mais temido e mais difícil de ser realizado.

A literatura, principalmente a internacional, corrobora tais achados ao sugerir que existe uma imposição construída pela sociedade de que os homens devem ser fortes, sendo sugerido que, por essa razão, os homens temem revelar que estão doentes e ou que têm cuidados com sua saúde, pois temem o julgamento social e a perda da masculinidade (Boysen, 2017; Gomes et al., 2011; Mascayano et al., 2016). Tal fato é ainda mais acentuado quando o homem é acometido por doenças psiquiátricas (Boysen, 2017; Mascayano et al., 2016). Segundo relatos de profissionais da área da saúde, além das dificuldades estruturais que contribuem para a baixa procura dos homens aos serviços de saúde, a exposição das dificuldades masculinas provoca medo e vergonha nessa população, sendo entraves significativos para uma atitude ligada à prevenção de saúde (Silveira et al., 2017).

Contudo existem relatos de homens com câncer de que nada é mais impactante do que receber esse diagnóstico, o qual costuma ser permeado por sentimentos ligados ao medo, desesperança, angústia e tristeza. Além de arrependimentos, normalmente associados à busca tardia por serviços de saúde (Modena, Martins, Gazzinelli, Almeida, \& Schall, 2014). Nota-se que, embora os homens tenham consciência da importância da prevenção em saúde, a obrigatoriedade de demonstração de força e poder socialmente construída não dá ao homem o direito de revelar suas vulnerabilidades, já que o modelo imposto é de um ser potente, indestrutível, não sendo permitido chorar ou entrar em contato com a suas emoções, nem expressar seus medos ou ansiedades (Gomes et al., 2011; Viana et al., 2015).

\section{Categoria 3 - Valor do Trabalho na Vida dos Homens}

Os artigos 0, 1, 2 3, 6, 7 e 8 apresentam a importância do trabalho na vida dos homens, sendo compreendido pelos homens como uma forma de demonstrar seu valor, sua utilidade e sua masculinidade. A impossibilidade de trabalhar é assustadora para alguns homens, em especial devido ao risco de desemprego. O horário de funcionamento das unidades de saúde também foi apontado como um impeditivo para a realização do autocuidado.

0 artigo 1 revela que o adoecimento está relacionado à impossibilidade de trabalhar e, para os homens, exercer uma atividade dá sentido à sua masculinidade. Um dos participantes (O. M.) relatou "Eu não podia trabalhar como eu queria", como um dos principais problemas de sua doença (câncer).

Alguns participantes relataram: "Primeiro lugar, infelizmente, a gente homem se dedica muito ao trabalho, sustentar a família, ai se preocupa muito com o bem-estar de manter a 
casa. Ele acha que não precisa ir ao médico. A realidade é essa" (H9) (artigo 2). "A mente do homem é mais trabalho é mais a parte financeira" (Gilson) (artigo 3). "Só estou aqui porque não estou conseguindo trabalhar" (A20) (artigo 0).

Nos artigos 0, 2, 3, 6 o trabalho aparece como grande valor de vida da população masculina em detrimento dos cuidados com a própria saúde. A busca por atendimento em saúde requer, muitas vezes, que o sujeito falte ao trabalho, sendo mais um fator que dificulta o acesso da população masculina ao sistema de saúde. Outros estudos corroboram essa visão ao expor que diversos homens argumentam que não é possível realizar exames de prevenção porque não dispõem do tempo necessário para realizar tal atividade, visto que o horário em que os serviços de saúde funcionam coincide com o horário comercial no qual ele desenvolve a sua atividade profissional, sendo um fator impeditivo na busca preventiva de saúde (Costa \& Moura, 2013; Santos et al., 2017).

Observa-se assim que o trabalho ocupa um espaço muito importante na vida dos indivíduos, principalmente quando falamos do gênero masculino, há significado no exercício do ofício evidenciado pelo relato do participante (O.M.) que relembrou, em vários momentos, sua vontade, mas impossibilidade para trabalhar (artigo 1). O trabalho costuma ser o motivo pelo qual os homens buscam o atendimento nos serviços de saúde, porém, normalmente isso ocorre quando as doenças já estão em estágios avançados, causando limitações físicas que afetam sua rotina de trabalho (Sanches, Cutolo, Soares, \& Silva, 2010; Amaral et al., 2017; Lemos et al., 2017).

Um estudo anterior corrobora a visão do participante O.M. (artigo 1) ao perceber que homens com diagnóstico de doença oncológica costumam apontar que a doença implica necessidade de afastamento do trabalho em função das limitações físicas e tratamento do câncer. Algumas vezes, esses indivíduos estão mais preocupados com as mudanças em suas rotinas do que com as possíveis consequências de sua condição clínica (Modena et al., 2014).

Outra situação importante a ser considerada está relacionada ao fato de que homens têm dificuldade de informar no trabalho que adoeceram, especialmente quando são doenças crônicas (Sanches et al., 2010; Viana et al., 2015). Além disso, é comum que os homens, entre os 20-59 anos, apresentem agressividade e impaciência quando precisam esperar ou não conseguem ser atendidos. É comum que usem essa demora de atendimento como uma desculpa para não buscarem tratamento em uma segunda ocasião (Gomes et al., 2011).

\section{Considerações Finais}

O presente estudo teve a intenção de verificar os fatores relacionados ao processo de autocuidado e adoecimento dos homens. A realização desta Revisão Integrativa da Literatura Nacional permitiu transitar por temáticas pertinentes ao público masculino brasileiro, o qual notadamente está distante de uma atitude preventiva em relação à própria saúde. Embora a PNAISH exista desde 2009, foi muito pouco citada pelos participantes dos estudos, o que pode demonstrar baixa efetividade junto ao público a que se destina, necessitando que seja mais bem divulgada e ampliada, levando em consideração as opiniões e a cultura dos homens residentes em diferentes regiões do país. 
Se a sociedade permanecer compreendendo o autocuidado como uma ação predominantemente feminina, o homem continuará agindo pela via da visão curativa de saúde, pois são permeados por sentimentos de medo, constrangimento e impotência, os quais os afastam dos comportamentos preventivos direcionados ao autocuidado.

Outro fator bastante significativo é a importância que o trabalho ocupa na vida dos homens, sendo considerada por eles como atividade prioritária em suas vidas. A atividade profissional é um dos pilares em que o homem exerce o seu poder pessoal, a sua criatividade, capacidade de ação, tomada de decisões, nas quais é solicitado a resolver situações. É de onde vem a sustentação financeira para si e eventualmente para outros membros da família. Contudo o trabalho acaba sendo priorizado pelos homens mesmo em casos de adoecimento, sendo negligenciada a busca por serviços de saúde até o desenvolvimento de sintomas que impossibilitem a realização de atividades laborais.

Em linhas gerais, os resultados encontrados apontam que a saúde integral da população masculina não é preventiva, não tem hábitos de autocuidado e parece estar perdida em um mundo distante do contemporâneo, o qual passa a demandar outro tipo de comportamento, um novo tipo de homem. Supõe-se então que essa população continuará aumentando nos índices de morbimortalidade até que consigam realizar avanços e mudanças comportamentais eficazes na direção que contemple aspectos de prevenção e autocuidado.

Contudo destaca-se que a responsabilidade não é somente dos homens, mas da sociedade como um todo, visto que é necessária a superação da cultura machista patriarcal. Além disso, o governo também pode auxiliar esta população através do desenvolvimento ou aprimoramento de políticas públicas já existentes, as quais devem considerar o meio sociocultural no qual os homens, nas mais diversas regiões de nosso país, estão inseridos. É primordial que o atendimento seja diferenciado dentro de cada realidade cultural, e não universal e idêntico em todas essas regiões.

Em relação às limitações deste estudo, destaca-se a utilização de apenas três bases de dados. Além disso, percebe-se que, de modo geral, ainda são escassas as publicações científicas brasileiras para o público masculino, fortalecendo a noção de certa invisibilidade para as questões de saúde dessa população. Tal limitação também dificultou a localização de estudos os quais pudessem ser utilizados para discussão dos resultados. Embora aparentemente exista, na literatura, um consenso sobre a falta de autocuidado da população masculina e os motivos que contribuem para que isso ocorra, ele se baseia na visão de poucos estudos.

Observou-se ainda que apenas um dos estudos incluídos nesta revisão foi realizado por psicólogos, demonstrando pouco interesse da psicologia nacional em estudar questões relacionadas à saúde do homem. Sendo assim, sugere-se que novos estudos, especialmente advindos da Psicologia, possam contribuir com intervenções que auxiliem os homens a buscarem os serviços de saúde.

\section{Agradecimentos}

Este estudo foi financiado em parte pelo Conselho Nacional de Desenvolvimento Científico e Tecnológico (CNPq) - código de financiamento 001. 


\section{Referências}

Amaral, D. E. D. D., Muniz, R. M., Cardoso, D. H., Noguez, P. T., Fagundes, R. F., \& Viegas, A. C. (2017). Câncer de mama masculino: o contexto do sobrevivente. Revista de Enfermagem da UFPE, 11(5), 1783-1790. doi: 10.5205/reuol.11077-98857-1-SM.1105201705

Backes, M. T. S., Rosa, L. M. D., Fernandes, G. C. M., Becker, S. G., Meirelles, B. H. S., \& Santos, S. M. D. A. D. (2009). Conceitos de saúde e doença ao longo da história sob o olhar epidemiológico e antropológico. Revista de Enfermagem da UERJ, 17(1), 111-117. Disponível em http://www.facenf.uerj.br/v17n1/v17n1a21.pdf

Batista, V. C. L., Ribeiro, L. C. C., Ribeiro, C. D. A. L., Paula, F. A., \& Araújo, A. (2016). Avaliação dos atributos da atenção primária à saúde segundo os profissionais de saúde da família. SANARE - Revista de Políticas Públicas, 15(02), 87-93. doi: 10.1016/S0032-3861(02)00774-7

Boff, L. (2014). Saber cuidar- Ética do humano, compaixão pela terra (20a ed.). Petrópolis: Editora Vozes.

Borges, L. M., \& Seidl, E. M. F. (2013). Efeitos de intervenção psicoeducativa sobre a utilização de serviços de saúde por homens idosos. Interface-Comunicação, Saúde, Educação, 17(47), 777-788. doi: 10.1590/S1414-32832013005000024

Boysen, G. A. (2017). Explaining the relation between masculinity and stigma toward mental illness: The relative effects of sex, gender, and behavior. Stigma and Health, 2(1), 66-79. doi: $10.1037 /$ sah0000041

Brasil. Ministério da Saúde (1997). Saúde da família: Uma estratégia para reorientação do modelo assistencial. Brasília: Ministério da Saúde. Disponível em http://bvsms.saude.gov. $\mathrm{br} / \mathrm{bvs} /$ publicacoes/cd09_16.pdf

Brasil. Ministério da Saúde (2009). Política nacional de atenção integral à saúde do homem. Brasília: Ministério da Saúde. Disponível em http://bvsms.saude.gov.br/bvs/publicacoes/ politica_nacional_atencao_saude_homem.pdf

Costa, T. B., \& Moura, V. L. F. (2013). O significado do toque da próstata para o homem: Enfermeiro na promoção da saúde. Revista de Pesquisa: Cuidado é Fundamental Online, 5(4), 537-564. doi: 10.9789/2175-5361.2013v5n4p537

Galvão, M. T. D. R. L., \& Janeiro, J. M. D. S. V. (2013). O autocuidado em enfermagem: Autogestão, automonitorização e gestão sintomática como conceitos relacionados. Revista Mineira de Enfermagem, 17(1), 226-236. doi: 10.5935/1415-2762.20130019

Gomes, R., Moreira, M. C. N., Nascimento, E. F. D., Rebello, L. E. F. D. S., Couto, M. T., \& Schraiber, L. B. (2011). Os homens não vêm! Ausência e/ou invisibilidade masculina na atenção primária. Ciência \& Saúde Coletiva, 16(suppl 1), 983-992. doi: 10.1590/S1413-81232011000700030

Hemmi, A. P. A., da Conceição, J. A., \& Santos, D. D. M. (2015). Representações sociais de homens sobre saúde e doença: Contribuições para o cuidado. Revista de Enfermagem do Centro-Oeste Mineiro, 5(1), 1457-1468. doi: 10.19175/recom.v0i0.642

Instituto Brasileiro de Geografia e Estatística (2018). Projeção da população do Brasil e das Unidades da Federação. Disponível em https://www.ibge.gov.br/apps/populacao/ projecao/

Lemos, A. P., Ribeiro, C., Fernandes, J., Bernardes, K., \& Fernandes, R. (2017). Saúde do homem: os motivos da procura dos homens pelos serviços de saúde. Revista de Enfermagem da UFPE, 11(11), 4546-4553. doi: 10.5205/reuol.11138-99362-1-SM.1111sup201714 
Lima, B., Martins, E. R. D. C., Ramos, R. C. D. A., Costa, C. M. A., Clos, A. C., \& Ferreira, J. I. C. (2014). Os meios alternativos de comunicação como ferramenta para a prevenção do câncer de próstata. Revista de Enfermagem da UERJ, 22(5), 656-662. doi: 10.12957/ reuerj.2014.15669

Mascayano, F., Tapia, T., Schilling, S., Alvarado, R., Tapia, E., Lips, W., \& Yang, L. H. (2016). Stigma toward mental illness in Latin America and the Caribbean: A systematic review. Revista Brasileira de Psiquiatria, 38(1), 73-85. doi: 10.1590/1516-4446-2015-1652

Medeiros, P. F., \& Guareschi, N. M. F. (2009). Políticas públicas de saúde da mulher: A integralidade em questão. Revista Estudos Feministas, 17(1), 31-48. doi: 10.1590/ S0104-026X2009000100003

Mendes, K. D. S., Silveira, R. C. C. P., \& Galvão, C. M. (2008). Revisão integrativa: Método de Pesquisa para a incorporação de evidências na saúde e na enfermagem. Texto \& Contexto em Enfermagem, 17(4),758-764. doi: 10.1590/S0104-07072008000400018

Modena, C. M., Martins, A. M., Gazzinelli, A. P., Almeida, S. S. L., Schall, V. T. (2014). Câncer e masculinidades: Sentidos atribuídos ao adoecimento e ao tratamento oncológico. Temas em Psicologia, 22(1), 67-78. doi: 10.9788/TP2014.1-06

Moreira, M. A., \& Carvalho, C. N. (2016). Atenção Integral à Saúde do Homem: Estratégias utilizadas por enfermeiras(os) nas Unidades de Saúde da Família do interior da Bahia. Saúde \& Transformação Social, 7(3), 121-132. Disponível em http://incubadora.periodicos. ufsc.br/index.php/saudeetransformacao/article/view/3660

Oliveira, V. B. C. A., \& Veríssimo, M. D. L. Ó. R. (2015). Children's health care assistance according to their families: A comparison between models of Primary Care. Revista da Escola de Enfermagem, 49(1), 30-36. doi: 10.1590/S0080-623420150000100004

Sanches, E. N., Cutolo, L. R. A., Soares, P., \& Silva, R. M. (2010). Organização do trabalho, sintomatologia dolorosa e significado de ser portador de LER/DORT. Psicologia Argumento, 28(63), 313-324. Disponível em https://periodicos.pucpr.br/index.php/ psicologiaargumento/article/view/20207

Santos, E. M., Figueredo, G. A., Mafra, A. L. S., Reis, H. F. T., Louzado, J. A., \& Santos, G. M. (2017). Saúde dos homens na percepção de enfermeiros da estratégia saúde da família. Revista de APS, 20(2), 231-238. Disponível em https://aps.uff.emnuvens.com.br/aps/ article/view/3090

Silva, A. M. M., Mambrini, J. V. M., Peixoto, S. V., Malta, D. C., \& Lima-Costa, M. F. (2017). Uso de serviços de saúde por idosos brasileiros com e sem limitação funcional. Revista de Saúde Pública, 51(suppl 1), 1-9. doi: 10.1590/S1518-8787.2017051000243

Silveira, C. L. G., Melo, V. F. C. D., \& Barreto, A. J. R. (2017). Atenção à saúde do homem na atenção primária em saúde: Uma revisão integrativa. Revista de Enfermagem da UFPE, 11(3), 1528-1535. doi: 10.5205/reuol.10263-91568-1-RV.1103sup201727

Sousa, A. R., Queiroz, A. M., Florencio, R. M. S., Portela, P. P., Fernandes, J. D., \& Pereira, A. (2016). Homens nos serviços de Atenção Básica à Saúde: Repercussões da construção social das masculinidades. Revista Baiana de Enfermagem, 30(3), 117-129. doi: 10.18471/ rbe.v30i3.16054

Storino, L. P., Souza, K. V., \& Silva, K. L. (2013). Necessidades de saúde de homens usuários na atenção básica: Acolhimento e vínculo como potencializadores da integralidade. Escola de Enfermagem Anna Nery, 17(4): 638-645. doi: 10.5935/1414-8145.20130006 
Tomé, B., \& Lopes, F. (2012). Quem fala do quê nas notícias de saúde do público em 2009: Uma análise crítica. Estudos em Jornalismo e Mídia, 9(2). 326-339. doi: 10.5007/1984-6924.2012v9n2p32 6

Vasconcelos, L. B., \& Frota, M. T. E. (2018). Saúde do homem na atenção primária : Relato de experiência. Cadernos da Escola de Saúde Pública do Ceará, 12(1), 116-129. Disponível em http://cadernos.esp.ce.gov.br/index.php/cadernos/article/view/137

Viana, M. E. R., Costa, L. M. C., Santos, R. M., Anjos, D. S. (2015). O cuidado à saúde da população masculina em tempos de Política Nacional de Atenção Integral à Saúde do Homem: O que eles falam. Cultura de los Cuidados,19(41), 135-146. doi: 10.14198/ cuid.2015.41.16

Wynter, K., Wilson, N., Thean, P., Bei, B., \& Fisher, J. (2018). Psychological distress, alcohol use, fatigue, sleepiness, and sleep quality: An exploratory study among men whose partners are admitted to a residential early parenting service. Australian Psychologist, 1-8. doi:10.1111/ap.12348

Xavier, S. Q., Ceolin, T., Guanilo, M. E. E., \& Mendieta, M. C. (2015). Concepção de saúde e autocuidado pela população masculina de uma Unidade Básica de Saúde. Revista Electrónica Trimestral de Enfermaria,7(2), 2372-2382. doi: 10.9789/2175-5361.2015. v7i2.2372-2382

Recebido: 21/02/2019

Última revisão: 24/04/2019

Aceite final: $24 / 04 / 2019$

\section{Sobre os autores:}

Luis Henrique Costa Garcia - Graduado em Psicologia pela Universidade Luterana do Brasil, Gravataí. E-mail: Icostagarcia@gmail.com, Orcid: http://orcid.org/0000-0003-4897-1415

Nicolas de Oliveira Cardoso - Mestrando em Psicologia Clínica na Pontifícia Universidade Católica do Rio Grande do Sul. Graduado em Psicologia pela Universidade Luterana do Brasil. E-mail: nicolas.deoliveira@hotmail.com, Orcid: http://orcid.org/0000-0002-1555-1409

Cláudia Maria Canestrine do Nascimento Bernardi - Doutora em Educação pela Pontifícia Universidade Católica do Rio Grande do Sul. Mestre em Educação pela Universidade do Vale do Rio dos Sinos (UNISINOS). Especialista em Educação e Graduada em Psicologia pela Universidade Católica de Pelotas (UFPel). Professora da Faculdade São Francisco de Assis. E-mail: claudia.nb@terra.com.br, Orcid: http://orcid.org/0000-0001-7648-2219 
